\title{
A transmission problem for the Timoshenko system
}

\author{
C.A. RAPOSO ${ }^{1}$, W.D. BASTOS ${ }^{2}$ and M.L. SANTOS $^{3}$ \\ ${ }^{1}$ Department of Mathematics, UFSJ, Praça Frei Orlando, 170 \\ 36307-352 São João del-Rei, MG \\ ${ }^{2}$ Department of Mathematics, UNESP, Rua Cristovão Colombo, 2265 \\ 15054-000 São José do Rio Preto, SP \\ ${ }^{3}$ Department of Mathematics, UFPA, Rua do Una, 156 - 66113-200 Pará, PA \\ E-mails: raposo@ufsj.edu.br / waldemar@ibilce.unesp.br / mlsantos@ufpa.br
}

\begin{abstract}
In this work we study a transmission problem for the model of beams developed by S.P. Timoshenko [10]. We consider the case of mixed material, that is, a part of the beam has friction and the other is purely elastic. We show that for this type of material, the dissipation produced by the frictional part is strong enough to produce exponential decay of the solution, no matter how small is its size. We use the method of energy to prove exponential decay for the solution.
\end{abstract}

Mathematical subject classification: $35 \mathrm{~J} 55,35 \mathrm{~J} 77,93 \mathrm{C} 20$.

Key words: transmission, Timoshenko, beams, exponential decay, frictional damping.

\section{Introduction}

The transverse vibration of a beam is mathematically described by a system of two coupled differential equations given by

$$
\begin{array}{ll}
\rho u_{t t}-\left(K\left(u_{x}+\psi\right)\right)_{x}=0, & \text { in }(0, L) \times(0, \infty), \\
I_{\rho} \psi_{t t}-\left(E I \psi_{x}\right)_{x}+K\left(u_{x}+\psi\right)=0, & \text { in }(0, L) \times(0, \infty) .
\end{array}
$$

Here, $L$ is the length of the beam in its equilibrium position, $t$ is the time variable and $x$ is the space coordinate along the beam. The function $u=u(x, t)$ 
is the transverse displacement of the beam and $\psi=\psi(x, t)$ is the rotation angle of a filament of the beam. The coefficients $\rho, I_{\rho}, E, I$ and $K$ are the mass per unit length, the polar moment of inertia of a cross section, Young's modulus of elasticity, the moment of inertia of a cross section and the shear modulus respectively. We denote $\rho_{1}=\rho, \rho_{2}=I_{\rho}, b=E I, k=K$ and we obtain directly from (1.1) the following system

$$
\begin{array}{ll}
\rho_{1} u_{t t}-k\left(u_{x}+\psi\right)_{x}=0, & \text { in }(0, L) \times(0, \infty), \\
\rho_{2} \psi_{t t}-b_{2} \psi_{x x}+k\left(u_{x}+\psi\right)=0, & \text { in }(0, L) \times(0, \infty) .
\end{array}
$$

The mathematical model describing the vibrations of beam with fixed extremities is formed by the system (1.2), boundary conditions

$$
u(0, t)=u(L, t)=\psi(0, t)=\psi(L, t)=0, \quad t>0,
$$

and initial data

$$
u(\cdot, 0)=\phi_{0}, \quad u_{t}(\cdot, 0)=\phi_{1}, \quad \psi(\cdot, 0)=\psi_{0}, \quad \psi_{t}(\cdot, 0)=\psi_{1}, \quad \text { in }(0, L) .
$$

If friction is taken into account, the system (1.2) becomes

$$
\begin{array}{ll}
\rho_{1} u_{t t}-k\left(u_{x}+\psi\right)_{x}+\alpha u_{t}=0, & \text { in }(0, L) \times(0, \infty), \\
\rho_{2} \psi_{t t}-b_{2} \psi_{x x}+k\left(u_{x}+\psi\right)+\beta \psi_{t}=0, & \text { in }(0, L) \times(0, \infty),
\end{array}
$$

where $\alpha$ and $\beta$ are positive constants (we assume $\alpha=\beta=1$ ). The terms $\alpha u_{t}$ and $\beta \psi_{t}$ represent the attrition acting in the vertical vibrations and in the angle of rotation of the filaments of the beam, respectively.

Dissipative properties associated to the system (1.3) have been studied by several authors by considering dissipative mechanism of frictional or viscoelastic type. The frictional dissipation, obtained by introduction of a frictional mechanism acting on the entire domain or on the boundary, was studied in [7, 8, 9]. The viscoelastic dissipation, given by a memory effect, was considered in [2] and [6].

An interesting problem comes out when the dissipation acts only on a part of the domain. In the present paper we consider a frictional mechanism acting only on the part of the domain given by $0 \leq x \leq L_{0}$ with $0<L_{0}<L$. We prove that for every $L_{0}$ the energy of the system decays exponentially to zero as time 
goes to infinity. In other words, our result states that dissipative properties of the system are transferred to the whole beam and stabilizes the system. The main result of this paper is Theorem 2 and its corollary, both in section 5 .

The mathematical model which deals with this situation is called a transmission problem. From the mathematical point of view a transmission problem consist of an initial and boundary value problem for a hyperbolic equation for which the corresponding elliptic operator has discontinuous coefficients. Hence, we can not expect to have regular solutions in the role domain. In the next section we establish the transmission problem and define appropriately the notion of solution considered. We use $H^{m}$ and $L^{p}$ to denote the usual Sobolev and Lebesgue spaces [1].

\section{The transmission problem}

In this section we describe precisely the transmission problem treated in the paper and establish existence and regularity of solution. We begin by introducing the notation

$$
\begin{aligned}
\rho_{j}(x) & =\left\{\begin{array}{lll}
\rho_{j}^{1}, & \text { if } & x \in\left(0, L_{0}\right) \\
\rho_{j}^{2}, & \text { if } & x \in\left(L_{0}, L\right)
\end{array}\right\}, \\
k(x) & =\left\{\begin{array}{lll}
k_{1}, & \text { if } & x \in\left(0, L_{0}\right) \\
k_{2}, & \text { if } & x \in\left(L_{0}, L\right)
\end{array}\right\}, \\
b(x) & =\left\{\begin{array}{lll}
b_{1}, & \text { if } & x \in\left(0, L_{0}\right) \\
b_{2}, & \text { if } & x \in\left(L_{0}, L\right)
\end{array}\right\}, \\
\alpha & =\left\{\begin{array}{lll}
1, & \text { if } & x \in\left(0, L_{0}\right) \\
0, & \text { if } & x \in\left(L_{0}, L\right)
\end{array}\right\}, \\
\beta & =\left\{\begin{array}{lll}
1, & \text { if } & \left.x \in\left(0, L_{0}\right)\right] \\
0, & \text { if } & x \in\left(L_{0}, L\right)
\end{array}\right\}, \\
u(x, t) & =\left\{\begin{array}{lll}
u(x, t), & \text { if } & x \in\left(0, L_{0}\right) \times(0, \infty) \\
v(x, t), & \text { if } & x \in\left(L_{0}, L\right) \times(0, \infty)
\end{array}\right\},
\end{aligned}
$$




$$
\psi(x, t)=\left\{\begin{array}{lll}
\psi(x, t), & \text { if } & x \in\left(0, L_{0}\right) \times(0, \infty) \\
\phi(x, t), & \text { if } & x \in\left(L_{0}, L\right) \times(0, \infty)
\end{array}\right\} .
$$

Using the notation above, model (1.3) can be written in the following form:

$$
\begin{array}{ll}
\rho_{1}^{1} u_{t t}-k_{1}\left(u_{x}+\psi\right)_{x}+u_{t}=0, & \text { in } \quad\left(0, L_{0}\right) \times(0, \infty), \\
\rho_{2}^{1} \psi_{t t}-b_{1} \psi_{x x}+k_{1}\left(u_{x}+\psi\right)+\psi_{t}=0, & \text { in }\left(0, L_{0}\right) \times(0, \infty), \\
\rho_{1}^{2} v_{t t}-k_{2}\left(v_{x}+\phi\right)_{x}=0, & \text { in }\left(L_{0}, L\right) \times(0, \infty), \\
\rho_{2}^{2} \phi_{t t}-b_{2} \phi_{x x}+k_{2}\left(v_{x}+\phi\right)=0, & \text { in }\left(L_{0}, L\right) \times(0, \infty),
\end{array}
$$

\section{Dissipative part}

$$
u(x), \quad \psi(x)
$$

\section{Elastic part}

$v(x), \phi(x)$
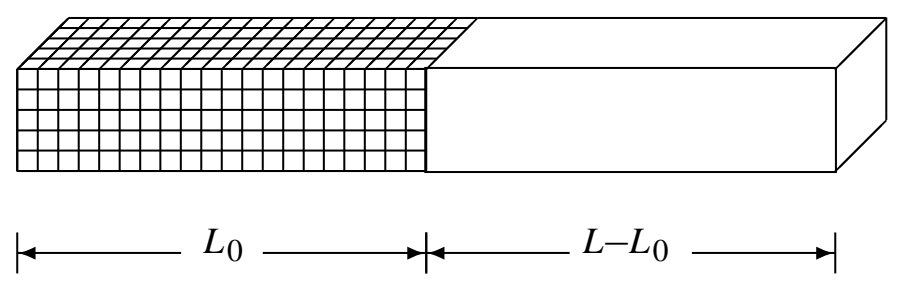

with boundary conditions,

$$
u(0, t)=v(L, t)=\psi(0, t)=\phi(L, t)=0, \quad t>0,
$$

transmission conditions,

$$
\begin{array}{ll}
k_{1} u\left(L_{0}, t\right)=k_{2} v\left(L_{0}, t\right), & \rho_{1}^{1} u_{t}\left(L_{0}, t\right)=\rho_{1}^{2} v_{t}\left(L_{0}, t\right), \\
k_{1} u_{x}\left(L_{0}, t\right)=k_{2} v_{x}\left(L_{0}, t\right), & k_{1} \psi\left(L_{0}, t\right)=k_{2} \phi\left(L_{0}, t\right), \\
\rho_{2}^{1} \psi_{t}\left(L_{0}, t\right)=\rho_{2}^{2} \phi_{t}\left(L_{0}, t\right), & b_{1} \psi_{x}\left(L_{0}, t\right)=b_{2} \phi_{x}\left(L_{0}, t\right),
\end{array}
$$

and initial data

$$
\begin{aligned}
& u(\cdot, 0)=u_{0}, \quad u_{t}(\cdot, 0)=u_{1}, \quad \psi(\cdot, 0)=\psi_{0}, \quad \psi_{t}(\cdot, 0)=\psi_{1}, \quad \text { in }\left(0, L_{0}\right), \\
& v(\cdot, 0)=v_{0}, \quad v_{t}(\cdot, 0)=v_{1}, \quad \phi(\cdot, 0)=\phi_{0}, \quad \phi_{t}(\cdot, 0)=\phi_{1}, \quad \text { in }\left(L_{0}, L\right) .
\end{aligned}
$$

We define the notion of weak solution to the system (2.1)-(2.6) as follows: 
Definition 1. Let $\mathcal{V}, \mathcal{H}^{m}$ and $\mathcal{L}^{2}$ be the spaces defined by

$$
\begin{aligned}
& \mathcal{V}=\left\{(\bar{w}, w) \in H^{1}\left(0, L_{0}\right) \times H^{1}\left(L_{0}, L\right) ; \quad \bar{w}(0)=w(L)=0, \quad \bar{w}\left(L_{0}\right)=w\left(L_{0}\right)\right\} \\
& \mathcal{H}^{m}=H^{m}\left(0, L_{0}\right) \times H^{m}\left(L_{0}, L\right) \quad \text { and } \quad \mathcal{L}^{2}=L^{2}\left(0, L_{0}\right) \times L^{2}\left(L_{0}, L\right) .
\end{aligned}
$$

We say that $(u, v, \psi, \phi)$ is a weak solution to the problem (2.1)-(2.6) if for every $(\bar{w}, w) \in H_{0}^{1}\left(0, T ; \mathcal{H}^{2} \cap \mathcal{V}\right)$ we have:

$$
\begin{aligned}
& \rho_{1}^{1} \int_{0}^{L_{0}} u_{t}(x, T) \bar{w}(x, T) d x-\rho_{1}^{1} \int_{0}^{L_{0}} u_{t}(x, 0) \bar{w}(x, 0) d x \\
& \quad+\rho_{1}^{2} \int_{L_{0}}^{L} v_{t}(x, T) w(x, T) d x-\rho_{1}^{2} \int_{L_{0}}^{L} v_{t}(x, 0) w(x, 0) d x \\
& \quad-\rho_{1}^{1} \int_{0}^{T} \int_{0}^{L_{0}} u_{t}(x, t) \bar{w}_{t}(x, t) d x d t-\rho_{1}^{2} \int_{0}^{T} \int_{L_{0}}^{L} v_{t}(x, t) w_{t}(x, t) d x d t \\
& \quad+\int_{0}^{T} \int_{L_{0}}^{L} v_{t}(x, t) w_{x}(x, t) d x d t+\int_{0}^{T} \int_{0}^{L_{0}} k_{1}\left(u_{x}+\psi\right)(x, t) \bar{w}_{x}(x, t) d x d t \\
& \quad+\int_{0}^{T} \int_{L_{0}}^{L} k_{2}\left(v_{x}+\phi\right)(x, t) w_{x}(x, t) d x d t=0
\end{aligned}
$$

and

$$
\begin{aligned}
& \rho_{1}^{2} \int_{0}^{L_{0}} \psi_{t}(x, T) \bar{w}(x, T) d x-\rho_{1}^{2} \int_{0}^{L_{0}} \psi_{t}(x, 0) \bar{w}(x, 0) d x \\
& \quad+\rho_{2}^{2} \int_{L_{0}}^{L} \phi_{t}(x, T) w(x, T) d x-\rho_{2}^{2} \int_{L_{0}}^{L} \phi_{t}(x, 0) w(x, 0) d x \\
& \quad-\rho_{2}^{1} \int_{0}^{T} \int_{0}^{L_{0}} \psi_{t}(x, t) \bar{w}_{t}(x, t) d x d t-\rho_{2}^{2} \int_{0}^{T} \int_{L_{0}}^{L} \phi_{t}(x, t) w_{t}(x, t) d x d t \\
& \quad+\int_{0}^{T} \int_{0}^{L_{0}} b_{1} \psi(x, t)_{x} \bar{w}(x, t) d x d t+\int_{0}^{T} \int_{0}^{L_{0}} \psi_{t}(x, t) \bar{w}_{x}(x, t) d x d t \\
& \quad+\int_{0}^{T} \int_{0}^{L_{0}} k_{1}\left(u_{x}+\psi\right) \bar{w}(x, t) d x d t+b_{2} \int_{0}^{T} \int_{L_{0}}^{L} \phi_{x}(x, t) w_{x}(x, t) d x d t \\
& \quad+\int_{0}^{T} \int_{L_{0}}^{L} k_{2}\left(v_{x}+\phi\right)(x, t) w(x, t) d x d t=0 .
\end{aligned}
$$

The transmission problem for a single hyperbolic equation was studied by Dautray and Lions [3], who proved the existence and regularity of solutions for 
the linear problem. The existence and regularity of solutions to the transmission problem for the Timoshenko system is given in the following theorem:

Theorem 1. If $\left(u_{0}, v_{0}\right),\left(\psi_{0}, \phi_{0}\right) \in \mathcal{V}$ and $\left(u_{1}, v_{1}\right),\left(\psi_{1}, \phi_{1}\right) \in \mathcal{L}^{2}$, then there exists a unique weak solution $(u, v, \psi, \phi)$ to the system (2.1)-(2.6) satisfying:

$$
(u, v),(\psi, \phi) \in C(0, \infty ; \mathcal{V}) \cap C^{1}\left(0, \infty ; \mathcal{L}^{2}\right) .
$$

Moreover, if $\left(u_{0}, v_{0}\right),\left(\psi_{0}, \phi_{0}\right) \in \mathcal{H}^{2} \cap \mathcal{V}$ and $\left(u_{1}, v_{1}\right),\left(\psi_{1}, \phi_{1}\right) \in \mathcal{V}$, then the weak solution is a strong solution and satisfies

$$
(u, v),(\psi, \phi) \in C\left(0, \infty ; \mathcal{H}^{2} \cap \mathcal{V}\right) \cap C^{1}(0, \infty ; \mathcal{V}) \cap C^{2}\left(0, \infty ; \mathcal{L}^{2}\right)
$$

Proof. For the proof we proceed in a quite similar manner as in [3].

The total energy associated to the system is defined by

$$
\begin{aligned}
E(t)= & \frac{1}{2} \int_{0}^{L_{0}}\left\{\rho_{1}^{1}\left|u_{t}\right|^{2}+\rho_{2}^{1}\left|\psi_{t}\right|^{2}+b_{1}\left|\psi_{x}\right|^{2}+k_{1}\left|u_{x}+\psi\right|^{2}\right\} d x \\
& +\frac{1}{2} \int_{L_{0}}^{L}\left\{\rho_{1}^{2}\left|v_{t}\right|^{2}+\rho_{2}^{2}\left|\phi_{t}\right|^{2}+b_{2}\left|\phi_{x}\right|^{2}+k_{2}\left|v_{x}^{2}+\phi\right|^{2}\right\} d x .
\end{aligned}
$$

Next we prove that the total energy associated to the system is decreasing for every $t>0$.

Lemma 1. Let $(u, v, \psi, \phi)$ be the strong solution to the system (2.1)-(2.6), then

$$
\frac{d}{d t} E(t)=-\int_{0}^{L_{0}}\left|u_{t}\right|^{2} d x-\int_{0}^{L_{0}}\left|\psi_{t}\right|^{2} d x
$$

Proof. Multiplying (2.1) by $u_{t}$ and integrating by parts over the interval $\left(0, L_{0}\right)$, we get

$$
\begin{gathered}
\frac{d}{d t} \frac{\rho_{1}^{1}}{2} \int_{0}^{L_{0}}\left|u_{t}\right|^{2} d x=k_{1}\left(u_{x}\left(L_{0}\right)+\psi\left(L_{0}\right)\right) u_{t}\left(L_{0}\right) \\
\quad-k_{1} \int_{0}^{L_{0}}\left(u_{x}+\psi\right) u_{t x} d x-\int_{0}^{L_{0}}\left|u_{t}\right|^{2} d x
\end{gathered}
$$


Now, multiplying (2.2) by $\psi_{t}$ and integrating by parts over $\left(0, L_{0}\right)$ we obtain

$$
\begin{gathered}
\frac{d}{d t}\left\{\frac{\rho_{2}^{1}}{2} \int_{0}^{L_{0}}\left|\psi_{t}\right|^{2} d x+\frac{b_{1}}{2} \int_{0}^{L_{0}}\left|\psi_{x}\right|^{2} d x\right\}=b_{1} \psi_{x}\left(L_{0}\right) \psi_{t}\left(L_{0}\right) \\
-k_{1} \int_{0}^{L_{0}}\left(u_{x}+\psi\right) \psi_{t} d x-\int_{0}^{L_{0}}\left|\psi_{t}\right|^{2} d x
\end{gathered}
$$

Multiplying (2.3) by $v_{t}$ and integrating by parts on $\left(L_{0}, L\right)$, we get

$$
\frac{\rho_{1}^{2}}{2} \frac{d}{d t} \int_{L_{0}}^{L}\left|v_{t}\right|^{2} d x=-k_{2}\left(v_{x}+\phi\right)\left(L_{0}\right) v_{t}\left(L_{0}\right)-k_{2} \int_{L_{0}}^{L}\left(v_{x}+\phi\right) v_{t x} d x .
$$

Multiplying (2.4) by $\phi_{t}$ and integrating by parts on $\left(L_{0}, L\right)$ leads to

$$
\begin{aligned}
\frac{d}{d t}\left\{\frac{\rho_{2}^{2}}{2} \int_{L_{0}}^{L}\left|\phi_{t}\right|^{2} d x\right. & \left.+\frac{b_{2}}{2} \int_{L_{0}}^{L}\left|\phi_{x}\right|^{2} d x\right\}=-b_{2} \phi_{x}\left(L_{0}\right) \phi_{t}\left(L_{0}\right) \\
- & k_{2} \int_{L_{0}}^{L}\left(v_{x}+\phi\right) \phi_{t} d x
\end{aligned}
$$

Now observe that

$$
\frac{k_{1}}{2} \frac{d}{d t} \int_{0}^{L_{0}}\left|\phi_{x}^{1}+\psi^{1}\right|^{2} d x=k_{1} \int_{0}^{L_{0}}\left(\phi_{x}^{1}+\psi^{1}\right)\left(\phi_{x t}^{1}+\psi_{t}^{1}\right) d x
$$

and

$$
\frac{k_{2}}{2} \frac{d}{d t} \int_{L_{0}}^{L}\left|\phi_{x}^{2}+\psi^{2}\right|^{2} d x=k_{2} \int_{L_{0}}^{L}\left(\phi_{x}^{2}+\psi^{2}\right)\left(\phi_{x t}^{2}+\psi_{t}^{2}\right) d x .
$$

Summing up (2.7), (2.8), (2.9) and (2.10), and using (2.11) and (2.12) together with the hypothesis of transmission we obtain

$$
\frac{d}{d t} E(t)=-\int_{0}^{L_{0}}\left|u_{t}\right|^{2} d x-\int_{L_{0}}^{L}\left|\psi_{t}\right|^{2} d x
$$

\section{Technical lemmas}

Now we develop a series of technical results in order to facilitate the proof of the main result of the paper. We begin by constructing a functional $\mathcal{E}(t)$, equivalent to the energy functional, which satisfies $\mathcal{E}(t) \leq C \mathcal{E}(0), C<1$. In order to do so, we use some multiplier techniques (usually associated to control problems) 
and the following restrictions on the boundary conditions for the elastic part of the beam:

$$
\begin{aligned}
\left|v_{x}(L)\right|^{2} & \leq \frac{1}{2 L} \int_{L_{0}}^{L}\left|v_{x}\right|^{2} d x . \\
\left|\phi_{x}(L)\right|^{2} & \leq \frac{1}{2 L} \int_{L_{0}}^{L}\left|\phi_{x}\right|^{2} d x .
\end{aligned}
$$

\section{Lemma 2. Let us define}

$\mathcal{E}_{1}(t)=N_{1} E(t)+\int_{0}^{L_{0}} x\left(\rho_{1}^{1} u_{x} u_{t}+\rho_{2}^{1} \psi_{x} \psi_{t}\right) d x+\int_{L_{0}}^{L} x\left(\rho_{1}^{2} v_{x} v_{t}+\rho_{2}^{2} \phi_{x} \phi_{t}\right) d x$

Then

$$
\frac{d}{d t} \mathcal{E}_{1}(t) \leq-\frac{1}{4} \int_{0}^{L_{0}}\left[k_{1}\left|u_{x}\right|^{2}+b_{1}\left|\psi_{x}\right|^{2}\right] d x-\frac{1}{4} \int_{L_{0}}^{L}\left[k_{2}\left|v_{x}\right|^{2}+b_{2}\left|\phi_{x}\right|^{2}\right] d x .
$$

Proof. Multiply (2.1) by $x u_{x}$ and integrate by parts over $\left(0, L_{0}\right)$ to get

$$
\begin{aligned}
\frac{d}{d t} \int_{0}^{L_{0}} \rho_{1}^{1} x u_{t} u_{x} d x= & \frac{L_{0} \rho_{1}^{1}}{2}\left|u_{t}\left(L_{0}\right)\right|^{2}-\frac{\rho_{1}^{1}}{2} \int_{0}^{L_{0}}\left|u_{t}\right|^{2} d x \\
& +\frac{L_{0} k_{1}}{2}\left|u_{x}\left(L_{0}\right)\right|^{2}-\frac{k_{1}}{2} \int_{0}^{L_{0}}\left|u_{x}\right|^{2} d x \\
& +k_{1} \int_{0}^{L_{0}} x \psi_{x} u_{x} d x-\int_{0}^{L_{0}} x u_{t} u_{x} d x .
\end{aligned}
$$

Multiply (2.2) by $x \psi_{x}$ and integrate by parts over $\left(0, L_{0}\right)$ to obtain

$$
\begin{aligned}
\frac{d}{d t} \int_{0}^{L_{0}} \rho_{2}^{1} x \psi_{t} \psi_{x} d x= & \frac{L_{0} \rho_{2}^{1}}{2}\left|\psi_{t}\left(L_{0}\right)\right|^{2}-\frac{\rho_{2}^{1}}{2} \int_{0}^{L_{0}}\left|\psi_{t}\right|^{2} d x \\
& +\frac{L_{0} b_{1}}{2}\left|\psi_{x}\left(L_{0}\right)\right|^{2}-\frac{b_{1}}{2} \int_{0}^{L_{0}}\left|\psi_{x}\right|^{2} d x \\
& +\frac{L_{0} k_{1}}{2}\left|\psi\left(L_{0}\right)\right|^{2}-\frac{k_{1}}{2} \int_{0}^{L_{0}}|\psi|^{2} d x \\
& -k_{1} \int_{0}^{L_{0}} x \psi_{x} u_{x} d x-\int_{0}^{L_{0}} x \psi_{t} \psi_{x} d x
\end{aligned}
$$


Multiplying (2.3) by $x v_{x}$ and integrating by parts over $\left(L_{0}, L\right)$ leads to

$$
\begin{aligned}
\frac{d}{d t} \int_{L_{0}}^{L} \rho_{1}^{2} x v_{t} v_{x} d x= & -\frac{L_{0} \rho_{1}^{2}}{2}\left|v_{t}\left(L_{0}\right)\right|^{2}-\frac{\rho_{1}^{2}}{2} \int_{L_{0}}^{L}\left|v_{t}\right|^{2} d x \\
& +\frac{L k_{2}}{2}\left|v_{x}(L)\right|^{2}-\frac{L_{0} k_{2}}{2}\left|v_{x}\left(L_{0}\right)\right|^{2} \\
& -\frac{k_{2}}{2} \int_{L_{0}}^{L}\left|v_{x}\right|^{2} d x+k_{2} \int_{L_{0}}^{L} x \phi_{x} v_{x} d x .
\end{aligned}
$$

Now multiply (2.4) by $x \phi_{x}$ and integrate over $\left(0, L_{0}\right)$ to obtain

$$
\begin{aligned}
\frac{d}{d t} \int_{L_{0}}^{L} \rho_{2}^{2} x \phi_{t} \phi_{x} d x= & -\frac{L_{0} \rho_{2}^{2}}{2}\left|\phi_{t}\left(L_{0}\right)\right|^{2}-\frac{\rho_{2}^{2}}{2} \int_{L_{0}}^{L}\left|\phi_{t}\right|^{2} d x \\
& +\frac{L b_{2}}{2}\left|\phi_{x}(L)\right|^{2}-\frac{L_{0} b_{2}}{2}\left|\phi_{x}\left(L_{0}\right)\right|^{2} \\
& -\frac{b_{2}}{2} \int_{L_{0}}^{L}\left|\phi_{x}\right|^{2} d x-\frac{L_{0} k_{2}}{2}\left|\phi\left(L_{0}\right)\right|^{2} \\
& -\frac{k_{2}}{2} \int_{L_{0}}^{L}\left|\phi_{x}\right|^{2} d x-k_{2} \int_{L_{0}}^{L} x \phi_{x} v_{x} d x
\end{aligned}
$$

Summing up (3.3), (3.4), (3.5), (3.6), and making use of the hypothesis on the transmission, the punctual terms are canceled and we get

$$
\begin{aligned}
\frac{d}{d t} \int_{0}^{L_{0}} x\left(\rho_{1}^{1} u_{x} u_{t}+\rho_{2}^{1} \psi_{x} \psi_{t}\right) d x+\frac{d}{d t} \int_{L_{0}}^{L} x\left(\rho_{1}^{2} v_{x} v_{t}+\rho_{2}^{2} \phi_{x} \phi_{t}\right) d x \\
=\frac{L k_{2}}{2}\left|v_{x}(L)\right|^{2}+\frac{L b_{2}}{2}\left|\phi_{x}(L)\right|^{2}-\frac{k_{1}}{2} \int_{0}^{L_{0}}\left|u_{x}\right|^{2} d x-\int_{0}^{L_{0}} x u_{t} u_{x} d x \\
\quad-\frac{b_{1}}{2} \int_{0}^{L_{0}}\left|\psi_{x}\right|^{2} d x+\int_{0}^{L_{0}} x \psi_{t} \psi_{x} d x-\frac{k_{2}}{2} \int_{L_{0}}^{L}\left|v_{x}\right|^{2} d x-\frac{b_{2}}{2} \int_{L_{0}}^{L}\left|\phi_{x}\right|^{2} d x
\end{aligned}
$$

Now using (3.1), (3.2) and the Young's inequality [4] we obtain

$$
\begin{aligned}
& \frac{d}{d t} \int_{0}^{L_{0}} x\left(\rho_{1}^{1} u_{x} u_{t}+\rho_{2}^{1} \psi_{x} \psi_{t}\right) d x+\frac{d}{d t} \int_{L_{0}}^{L} x\left(\rho_{1}^{2} v_{x} v_{t}+\rho_{2}^{2} \phi_{x} \phi_{t}\right) d x \\
& \quad \leq \frac{k_{2}}{4} \int_{L_{0}}^{L}\left|v_{x}\right|^{2} d x-\frac{k_{2}}{2} \int_{L_{0}}^{L}\left|v_{x}\right|^{2} d x+\frac{b_{2}}{4} \int_{L_{0}}^{L}\left|\phi_{x}\right|^{2} d x-\frac{b_{2}}{2} \int_{L_{0}}^{L}\left|\phi_{x}\right|^{2} d x
\end{aligned}
$$




$$
\begin{aligned}
& -\frac{k_{1}}{2} \int_{0}^{L_{0}}\left|u_{x}\right|^{2} d x+\frac{k_{1}}{4} \int_{0}^{L_{0}}\left|u_{x}\right|^{2} d x-\frac{b_{1}}{2} \int_{0}^{L_{0}}\left|\psi_{x}\right|^{2} d x+\frac{b_{1}}{4} \int_{0}^{L_{0}}\left|\psi_{x}\right|^{2} d x \\
& +\bar{C}\left[\int_{0}^{L_{0}}\left|\psi_{t}\right|^{2} d x+\int_{0}^{L_{0}}\left|u_{t}\right|^{2} d x\right] .
\end{aligned}
$$

Now, if we define

$\mathcal{E}_{1}(t)=N_{1} E(t)+\int_{0}^{L_{0}} x\left(\rho_{1}^{1} u_{x} u_{t}+\rho_{2}^{1} \psi_{x} \psi_{t}\right) d x+\int_{L_{0}}^{L} x\left(\rho_{1}^{2} v_{x} v_{t}+\rho_{2}^{2} \phi_{x} \phi_{t}\right) d x$ and choose $N_{1}>\bar{C}$ we conclude

$$
\frac{d}{d t} \mathcal{E}_{1}(t) \leq-\frac{1}{4} \int_{0}^{L_{0}}\left[k_{1}\left|u_{x}\right|^{2}+b_{1}\left|\psi_{x}\right|^{2}\right] d x-\frac{1}{4} \int_{L_{0}}^{L}\left[k_{2}\left|v_{x}\right|^{2}+b_{2}\left|\phi_{x}\right|^{2}\right] d x
$$

It is worth noticing that the estimate above is important in two aspects. First, it recovers a part of energy with minus sign. Second, it will play a fundamental role in the next two lemmas controlling punctual terms which will come up in the search for other negative terms of the energy.

\section{Lemma 3. Define}

$$
\mathcal{E}_{2}(t)=N_{2} E(t)+\int_{0}^{L_{0}} \rho_{1}^{1} x u_{t}\left(u_{x}+\psi\right) d x .
$$

Then

$$
\begin{aligned}
\frac{d}{d t} \mathcal{F}_{2}(t) \leq & \frac{\rho_{1}^{1} L_{0}}{2}\left|u_{t}\left(L_{0}\right)\right|^{2}+\frac{k_{1} L_{0}}{2}\left|u_{x}\left(L_{0}\right)+\psi\left(L_{0}\right)\right|^{2} \\
& -C \int_{0}^{L_{0}}\left[\left|u_{t}\right|^{2}+\left|\psi_{t}\right|^{2}+\left|u_{x}+\psi\right|^{2}\right] d x
\end{aligned}
$$

Proof. Multiply $(2.1)$ by $x\left(u_{x}+\psi\right)$ and integrate by parts over $\left(0, L_{0}\right)$ to obtain

$$
\begin{aligned}
\frac{d}{d t} \int_{0}^{L_{0}} x \rho_{1}^{1}\left(u_{x}+\psi\right) u_{t}= & \frac{\rho_{1}^{1} L_{0}}{2}\left|u_{t}\left(L_{0}\right)\right|^{2}-\frac{\rho_{1}^{1} L_{0}}{2} \int_{0}^{L_{0}}\left|u_{t}\right|^{2} d x \\
& +\frac{k_{1} L_{0}}{2}\left|u_{x}\left(L_{0}\right)+\psi\left(L_{0}\right)\right|^{2}-\frac{k_{1} L_{0}}{2} \int_{0}^{L_{0}}\left|u_{x}+\psi\right|^{2} d x \\
& +\rho_{1}^{1} \int_{0}^{L_{0}} x u_{t} \psi_{t} d x-\int_{0}^{L_{0}} x u_{t}\left(u_{x}+\psi\right) d x
\end{aligned}
$$


Using Young's inequality, we get

$$
\begin{aligned}
& \frac{d}{d t} \int_{0}^{L_{0}} x \rho_{1}^{1}\left(u_{x}+\psi\right) u_{t} \leq \frac{\rho_{1}^{1} L_{0}}{2}\left|u_{t}\left(L_{0}\right)\right|^{2}+\frac{k_{1} L_{0}}{2}\left|u_{x}\left(L_{0}\right)+\psi\left(L_{0}\right)\right|^{2} \\
& \quad-\frac{k_{1} L_{0}}{4} \int_{0}^{L_{0}}\left|u_{x}+\psi\right|^{2} d x+\tilde{C} \int_{0}^{L_{0}}\left|u_{t}\right|^{2} d x+\tilde{C} \int_{0}^{L_{0}}\left|\psi_{t}\right|^{2} d x
\end{aligned}
$$

Now, defining

$$
\mathcal{E}_{2}(t)=N_{2} E(t)+\int_{0}^{L_{0}} \rho_{1}^{1} x u_{t}\left(u_{x}+\psi\right) d x
$$

we obtain

$$
\begin{gathered}
\frac{d}{d t} \mathcal{E}_{2}(t) \leq \frac{\rho_{1}^{1} L_{0}}{2}\left|u_{t}\left(L_{0}\right)\right|^{2}+\frac{k_{1} L_{0}}{2}\left|u_{x}\left(L_{0}\right)+\psi\left(L_{0}\right)\right|^{2} \\
-\frac{k_{1} L_{0}}{4} \int_{0}^{L_{0}}\left|u_{x}+\psi\right|^{2} d x+\left(\tilde{C}-N_{2}\right) \int_{0}^{L_{0}}\left|u_{t}\right|^{2} d x+\left(\tilde{C}-N_{2}\right) \int_{0}^{L_{0}}\left|\psi_{t}\right|^{2} d x .
\end{gathered}
$$

If we choose $N_{2}>\tilde{C}$ we conclude that there exists $\breve{C}>0$ such that

$$
\begin{gathered}
\frac{d}{d t} \mathcal{E}_{2}(t) \leq \frac{\rho_{1}^{1} L_{0}}{2}\left|u_{t}\left(L_{0}\right)\right|^{2}+\frac{k_{1} L_{0}}{2}\left|u_{x}\left(L_{0}\right)+\psi\left(L_{0}\right)\right|^{2} \\
-\frac{k_{1} L_{0}}{4} \int_{0}^{L_{0}}\left|u_{x}+\psi\right|^{2} d x-\breve{C} \int_{0}^{L_{0}}\left|u_{t}\right|^{2} d x-\breve{C} \int_{0}^{L_{0}}\left|\psi_{t}\right|^{2} d x,
\end{gathered}
$$

and then

$$
\begin{aligned}
\frac{d}{d t} \mathcal{F}_{2}(t) \leq & \frac{\rho_{1}^{1} L_{0}}{2}\left|u_{t}\left(L_{0}\right)\right|^{2}+\frac{k_{1} L_{0}}{2}\left|u_{x}\left(L_{0}\right)+\psi\left(L_{0}\right)\right|^{2} \\
& -C \int_{0}^{L_{0}}\left[\left|u_{t}\right|^{2}+\left|\psi_{t}\right|^{2}+\left|u_{x}+\psi\right|^{2}\right] d x .
\end{aligned}
$$

Lemma 4. Let $\mathcal{E}_{3}$ be defined as

$\mathcal{E}_{3}(t)=\frac{\rho_{1}^{2} 2 L^{2}}{\rho_{2}^{2}} \int_{L_{0}}^{L} x\left(\rho_{2}^{1} v_{x} v_{t}+\rho_{2}^{1} \phi_{x} \phi_{t}\right) d x+\int_{L_{0}}^{L}(x-L)\left[\rho_{1}^{2} v_{t}\left(v_{x}+\phi\right)\right] d x$.

Then

$$
\begin{aligned}
\frac{d}{d t} \mathcal{E}_{3}(t) \leq & \frac{\rho_{1}^{2}\left(L-L_{0}\right)}{2}\left|v_{t}\left(L_{0}\right)\right|^{2}+\frac{k_{2}\left(L-L_{0}\right)}{2}\left|v_{x}\left(L_{0}\right)+\phi\left(L_{0}\right)\right|^{2} \\
& -C \int_{0}^{L_{0}}\left[\left|v_{t}\right|^{2}+\left|\phi_{t}\right|^{2}+\left|v_{x}+\phi\right|^{2}\right] d x .
\end{aligned}
$$


Proof. Multiply (2.3) by $(x-L)\left(v_{x}+\phi\right)$ and integrate by parts over $\left(L_{0}, L\right)$ to get

$$
\begin{aligned}
\frac{d}{d t} \int_{L_{0}}^{L}(x-L)\left[\rho_{1}^{2} v_{t}\left(v_{x}+\phi\right)\right] d x= & \frac{\rho_{1}^{2}\left(L-L_{0}\right)}{2}\left|v_{t}\left(L_{0}\right)\right|^{2}-\frac{\rho_{1}^{2}}{2} \int_{0}^{L_{0}}\left|v_{t}\right|^{2} d x \\
& +\frac{k_{2}\left(L-L_{0}\right)}{2}\left|v_{x}\left(L_{0}\right)+\phi\left(L_{0}\right)\right|^{2} \\
& -\frac{k_{2}}{2} \int_{L_{0}}^{L}\left|v_{x}+\phi\right|^{2} d x \\
& +\rho_{1}^{2} \int_{L_{0}}^{L}(x-L) v_{t} \phi_{t} d x .
\end{aligned}
$$

Using Young's inequality we obtain

$$
\begin{aligned}
\frac{d}{d t} \int_{L_{0}}^{L}(x-L)\left[\rho_{1}^{2} v_{t}\left(v_{x}+\phi\right)\right] d x \leq & \frac{\rho_{1}^{2}\left(L-L_{0}\right)}{2}\left|v_{t}\left(L_{0}\right)\right|^{2} \\
& +\frac{k_{2}\left(L-L_{0}\right)}{2}\left|v_{x}\left(L_{0}\right)+\phi\left(L_{0}\right)\right|^{2} \\
& -\frac{\rho_{1}^{2}}{2} \int_{0}^{L_{0}}\left|v_{t}\right|^{2} d x+\frac{\rho_{1}^{2}}{2} \int_{0}^{L_{0}}\left|v_{t}\right|^{2} d x \\
& -\frac{k_{2}}{2} \int_{L_{0}}^{L}\left|v_{x}+\phi\right|^{2} d x+\frac{L^{2} \rho_{1}^{2}}{2} \int_{L_{0}}^{L}\left|\phi_{t}\right|^{2} d x .
\end{aligned}
$$

Now we set

$\mathcal{E}_{3}(t)=\frac{\rho_{1}^{2} 2 L^{2}}{\rho_{2}^{2}} \int_{L_{0}}^{L} x\left(\rho_{2}^{1} v_{x} v_{t}+\rho_{2}^{1} \phi_{x} \phi_{t}\right) d x+\int_{L_{0}}^{L}(x-L)\left[\rho_{1}^{2} v_{t}\left(v_{x}+\phi\right)\right] d x$ and verify that

$$
\begin{aligned}
\frac{d}{d t} \mathcal{E}_{3}(t) \leq & \frac{\rho_{1}^{2}\left(L-L_{0}\right)}{2}\left|v_{t}\left(L_{0}\right)\right|^{2}+\frac{k_{2}\left(L-L_{0}\right)}{2}\left|v_{x}\left(L_{0}\right)+\phi\left(L_{0}\right)\right|^{2} \\
& -\frac{k_{2}}{2} \int_{L_{0}}^{L}\left|v_{x}+\phi\right|^{2} d x-\frac{L^{2} \rho_{1}^{2} \rho_{1}^{2}}{\rho_{2}^{2}} \int_{L_{0}}^{L}\left|v_{t}\right|^{2} d x \\
& +\left(\frac{L^{2} \rho_{1}^{2}}{2}-L^{2} \rho_{1}^{2}\right) \int_{L_{0}}^{L}\left|\phi_{t}\right|^{2} d x .
\end{aligned}
$$


It follows then

$$
\begin{aligned}
\frac{d}{d t} \mathcal{E}_{3}(t) \leq & \frac{\rho_{1}^{2}\left(L-L_{0}\right)}{2}\left|v_{t}\left(L_{0}\right)\right|^{2}+\frac{k_{2}\left(L-L_{0}\right)}{2}\left|v_{x}\left(L_{0}\right)+\phi\left(L_{0}\right)\right|^{2} \\
& -C \int_{0}^{L_{0}}\left[\left|v_{t}\right|^{2}+\left|\phi_{t}\right|^{2}+\left|v_{x}+\phi\right|^{2}\right] d x .
\end{aligned}
$$

Observe that in the attempt to recover the total energy of the system with negative sign we introduced the lemmas 3 and 4 . Now we need to control them. It will be achieved with the aid of the next section.

\section{Compactness}

This section is dedicated to discuss the argument of compactness employed in the proof of the main result of the paper. First we introduce a notation; the symbol $\rightarrow$ is used to denote convergence in the norm of the Sobolev space $L^{\infty}$ as in [5]. For sake of completeness, we state the following result due to J.U. Kim.

Lemma 5. Let $\left(u^{k}\right)$ be a sequence of functions satisfying

$$
\begin{aligned}
& u^{k} \rightarrow u \quad \text { in } \quad L^{\infty}\left(0, T, H^{\beta}(0, L)\right), \\
& u_{t}^{k} \rightarrow u_{t} \quad \text { in } L^{2}\left(0, T, H^{\alpha}(0, L)\right),
\end{aligned}
$$

as $k \rightarrow \infty$, with $\alpha<\beta$. Then

$$
u^{k} \rightarrow u \quad \text { in } C\left([0, T], H^{r}(0, L)\right)
$$

for some $r<\beta$.

Proof. See [5].

Lemma 6 (Lemma of compactness). If we define

$$
\begin{aligned}
B\left(L_{0}, t\right)= & \frac{\rho_{1}^{1} L_{0}}{2}\left|u_{t}\left(L_{0}\right)\right|^{2}+\frac{k_{1} L_{0}}{2}\left|u_{x}\left(L_{0}\right)+\psi\left(L_{0}\right)\right|^{2} \\
& +\frac{\rho_{1}^{2}\left(L-L_{0}\right)}{2}\left|v_{t}\left(L_{0}\right)\right|^{2}+\frac{k_{2}\left(L-L_{0}\right)}{2}\left|v_{x}\left(L_{0}\right)+\phi\left(L_{0}\right)\right|^{2}
\end{aligned}
$$


then, for every $\eta>0$ there exists a constant $C_{\eta}>0$ independent of the initial data, such that

$$
\begin{aligned}
\int_{0}^{T} B\left(L_{0}, t\right) d t \leq & \eta \int_{0}^{T} E(t) d t+C_{\eta}\left\{\int_{0}^{T} \int_{0}^{L_{0}}\left[\left|u_{x}\right|^{2}+\left|\psi_{x}\right|^{2}\right] d x d t\right. \\
& \left.+\int_{0}^{T} \int_{L_{0}}^{L}\left[\left|v_{x}\right|^{2}+\left|\phi_{x}\right|^{2}\right] d x d t\right\}
\end{aligned}
$$

for every strong solution $(u, v, \psi, \phi)$ to the system (2.1)-(2.6) and sufficiently large $T$.

Proof. We use a contradiction argument. Define

$$
\begin{aligned}
B^{n}\left(L_{0}, t\right)= & \frac{\rho_{1}^{1} L_{0}}{2}\left|u_{t}^{n}\left(L_{0}\right)\right|^{2}+\frac{k_{1} L_{0}}{2}\left|u_{x}^{n}\left(L_{0}\right)+\psi^{n}\left(L_{0}\right)\right|^{2} \\
& +\frac{\rho_{1}^{2}\left(L-L_{0}\right)}{2}\left|v_{t}^{n}\left(L_{0}\right)\right|^{2}+\frac{k_{2}\left(L-L_{0}\right)}{2}\left|v_{x}^{n}\left(L_{0}\right)+\phi^{n}\left(L_{0}\right)\right|^{2} .
\end{aligned}
$$

Suppose that there exists a sequence of initial data $\left(u_{0}^{n}, \psi_{0}^{n}\right) \in \mathcal{H}^{2} \cap \mathcal{V}, \quad\left(v_{0}^{n}, \phi_{0}^{n}\right) \in \mathcal{H}^{2} \cap \mathcal{V}, \quad\left(u_{1}^{n}, \psi_{1}^{n}\right) \in \mathcal{V}, \quad\left(v_{1}^{n}, \phi_{1}^{n}\right) \in \mathcal{V}$, and a positive constant $\eta_{0}>0$ such that the corresponding solution $\left(u^{n}, \psi^{n}\right)$, $\left(v^{n}, \phi^{n}\right)$ of the problem

$$
\begin{array}{ll}
\rho_{1}^{1} u_{t t}^{n}-k_{1}\left(u_{x}^{n}+\psi^{n}\right)_{x}+u_{t}^{n}=0 & \text { in }\left(0, L_{0}\right) \times(0, \infty), \\
\rho_{2}^{1} \psi_{t t}^{n}-b_{1} \psi_{x x}^{n}+k_{1}\left(u_{x}^{n}+\psi^{n}\right)+\psi_{t}^{n}=0 & \text { in }\left(0, L_{0}\right) \times(0, \infty), \\
\rho_{1}^{2} v_{t t}^{n}-k_{2}\left(v_{x}^{n}+\phi^{n}\right)_{x}=0 & \text { in }\left(L_{0}, L\right) \times(0, \infty), \\
\rho_{2}^{2} \phi_{t t}^{n}-b_{2} \phi_{x x}^{n}+k_{2}\left(v_{x}^{n}+\phi^{n}\right)=0 & \text { in }\left(L_{0}, L\right) \times(0, \infty),
\end{array}
$$

with boundary conditions,

$$
u^{n}(0, t)=v^{n}(L, t)=\psi^{n}(0, t)=\phi^{n}(L, t)=0, \quad t>0,
$$

transmission conditions,

$$
\begin{array}{ll}
k_{1} u^{n}\left(L_{0}, t\right)=k_{2} v^{n}\left(L_{0}, t\right), & \rho_{1}^{1} u_{t}^{n}\left(L_{0}, t\right)=\rho_{1}^{2} v_{t}^{n}\left(L_{0}, t\right), \\
k_{1} u_{x}^{n}\left(L_{0}, t\right)=k_{2} v_{x}^{n}\left(L_{0}, t\right), & k_{1} \psi^{n}\left(L_{0}, t\right)=k_{2} \phi^{n}\left(L_{0}, t\right), \\
\rho_{2}^{1} \psi_{t}^{n}\left(L_{0}, t\right)=\rho_{2}^{2} \phi_{t}^{n}\left(L_{0}, t\right), & b_{1} \psi_{x}^{n}\left(L_{0}, t\right)=b_{2} \phi_{x}^{n}\left(L_{0}, t\right),
\end{array}
$$


and initial data

$$
\begin{array}{cccc}
u^{n}(\cdot, 0)=u_{0}^{n}, & u_{t}(\cdot, 0)=u_{1}^{n}, & \psi^{n}(\cdot, 0)=\psi_{0}^{n}, & \psi_{t}^{n}(\cdot, 0)=\psi_{1}^{n} \quad \text { in } \quad\left(0, L_{0}\right), \\
v^{n}(\cdot, 0)=v_{0}^{n}, & v_{t}^{n}(\cdot, 0)=v_{1}^{n}, & \phi^{n}(\cdot, 0)=\phi_{0}^{n}, & \phi_{t}^{n}(\cdot, 0)=\phi_{1}^{n} \quad \text { in } \quad\left(L_{0}, L\right),
\end{array}
$$

satisfies

$$
\int_{0}^{T} B^{n}\left(L_{0}, t\right) d t=1, \quad n \in \mathbf{N}
$$

and the following inequality

$$
\begin{aligned}
1> & \eta_{0} \int_{0}^{T} E^{n}(t) d t+n\left\{\int_{0}^{T} \int_{0}^{L_{0}}\left[\left|u_{x}^{n}\right|^{2}+\left|\psi_{x}^{n}\right|^{2}\right] d x d t\right. \\
& \left.+\int_{0}^{T} \int_{L_{0}}^{L}\left[\left|v_{x}^{n}\right|^{2}+\left|\phi_{x}^{n}\right|^{2}\right] d x d t\right\} .
\end{aligned}
$$

Then the integral

$$
\int_{0}^{T} E^{n}(t) d t \quad \text { is bounded for every } \quad n \in \mathbf{N},
$$

and also,

$$
\int_{0}^{T} \int_{0}^{L_{0}}\left[\left|u_{x}^{n}\right|^{2}+\left|\psi_{x}^{n}\right|^{2}\right] d x d t \rightarrow 0 \quad \text { as } \quad n \rightarrow \infty
$$

and

$$
\int_{0}^{T} \int_{L_{0}}^{L}\left[\left|v_{x}^{n}\right|^{2}+\left|\phi_{x}^{n}\right|^{2}\right] d x d t \rightarrow 0 \quad \text { as } \quad n \rightarrow \infty .
$$

Now we observe that $E^{n}(t)>0$ and that $\int_{0}^{T} E^{n}(t) d t$ is bounded. Hence $E^{n}(t)$ is bounded and we can take a subsequence of $\left(u^{n}, \psi^{n}\right),\left(v^{n}, \phi^{n}\right)$ (for which we use the same notations) such that

$$
\begin{array}{llll}
u^{n}-u & \text { in } & L^{\infty}\left(0, T, \mathcal{H}^{2} \cap \mathcal{V}\right), \\
\psi^{n}-\psi & \text { in } \quad L^{\infty}\left(0, T, \mathcal{H}^{2} \cap \mathcal{V}\right), \\
v^{n}-v & \text { in } \quad L^{\infty}(0, T, \mathcal{V}), \\
\phi^{n}-\phi & \text { in } \quad L^{\infty}(0, T, \mathcal{V}) .
\end{array}
$$

Applying the lemma 5 we conclude that for $r<1$

$$
\begin{array}{lllll}
u^{n} & \rightarrow & \text { in } & C\left([0, T] ; H^{r}\left(0, L_{0}\right)\right), \\
\psi^{n} & \rightarrow & \text { in } & C\left([0, T] ; H^{r}\left(0, L_{0}\right)\right), \\
v^{n} & \rightarrow & \text { in } & C\left([0, T] ; H^{r}\left(L_{0}, L\right)\right), \\
\phi^{n} & \rightarrow & \text { in } & C\left([0, T] ; H^{r}\left(L_{0}, L\right)\right) .
\end{array}
$$


It follows from (4.1) that

$$
\int_{0}^{T} B\left(L_{0}, t\right)=1
$$

We observe that the convergences (4.2) and (4.3) result in

$$
\begin{array}{lll}
u_{x}=0 & \text { almost everywhere in } & \left(0, L_{0}\right) \times(0, T), \\
\psi_{x}=0 & \text { almost everywhere in } & \left(0, L_{0}\right) \times(0, T), \\
v_{x}=0 & \text { almost everywhere in } & \left(L_{0}, L\right) \times(0, T), \\
\phi_{x}=0 & \text { almost every where in } & \left(L_{0}, L\right) \times(0, T) .
\end{array}
$$

Now, applying Poincare's inequality we obtain

$$
\begin{aligned}
\int_{0}^{T}\left|u\left(L_{0}\right)\right|^{2} d t & \leq c_{p}^{2} \int_{0}^{T} \int_{0}^{L_{0}}\left|u_{x}\right|^{2} d x d t=0, \\
\int_{0}^{T}\left|u_{x}\left(L_{0}\right)\right|^{2} d t & \leq c_{p}^{2} \int_{0}^{T} \int_{0}^{L_{0}}\left|u_{x x}\right|^{2} d x d t=0, \\
\int_{0}^{T}\left|\psi\left(L_{0}\right)\right|^{2} d t & \leq c_{p}^{2} \int_{0}^{T} \int_{0}^{L_{0}}\left|\psi_{x}\right|^{2} d x d t=0, \\
\int_{0}^{T}\left|v\left(L_{0}\right)\right|^{2} d t & \leq c_{p}^{2} \int_{0}^{T} \int_{L_{0}}^{L}\left|v_{x}\right|^{2} d x d t=0, \\
\int_{0}^{T}\left|v_{x}\left(L_{0}\right)\right|^{2} d t & \leq c_{p}^{2} \int_{0}^{T} \int_{L_{0}}^{L}\left|v_{x x}\right|^{2} d x d t=0, \\
\int_{0}^{T}\left|\phi\left(L_{0}\right)\right|^{2} d t & \leq c_{p}^{2} \int_{0}^{T} \int_{L_{0}}^{L}\left|\phi_{x}\right|^{2} d x d t=0 .
\end{aligned}
$$

This estimates implies

$$
\int_{0}^{T} B\left(L_{0}, t\right)=0
$$

which is a contradiction to (4.4). This completes the proof of the lemma.

We are now ready to prove the main result of this paper, that is, the exponential decay of the energy associated to the transmission problem for the Timoshenko System with frictional dissipation. This is the content of the next section.

\section{Exponential decay}

Theorem 2. Let $(u, \psi, v, \phi)$ be a strong solution to the transmission problem for the Timoshenko System defined by (2.1)-(2.6). Then there exist positive 
constants $C$ and $w$ such that

$$
E(t) \leq C E(0) e^{-\omega t}
$$

Proof. We start defining

$$
\mathcal{E}(t)=N_{3} \mathcal{E}_{1}(t)+\mathcal{E}_{2}(t)+\mathcal{E}_{3}(t) .
$$

It follows from lemmas 2, 3, and 4

$$
\begin{aligned}
\frac{d}{d t} \mathcal{E}(t) \leq & -C_{0} E(t)-C_{1} N_{3}\left\{\int_{0}^{L_{0}}\left[\left|u_{x}\right|^{2}+\left|\psi_{x}\right|^{2}\right] d x\right. \\
& \left.+\int_{L_{0}}^{L}\left[\left|v_{x}\right|^{2}+\left|\phi_{x}\right|^{2}\right] d x\right\} .+B\left(L_{0}, t\right) .
\end{aligned}
$$

Now, integrating this inequality over $(0, T)$ and using the Lemma of Compactness we obtain

$$
\begin{aligned}
\mathcal{E}(T)-\mathcal{E}(0) \leq- & C_{0} \int_{0}^{T} E(t) d t+\eta \int_{0}^{T} E(t) d t \\
& -C_{1} N_{3}\left\{\int_{0}^{T} \int_{0}^{L_{0}}\left[\left|u_{x}\right|^{2}+\left|\psi_{x}\right|^{2}\right] d x d t\right. \\
& \left.+\int_{0}^{T} \int_{L_{0}}^{L}\left[\left|v_{x}\right|^{2}+\left|\phi_{x}\right|^{2}\right] d x d t\right\} \\
+ & C_{\eta}\left\{\int_{0}^{T} \int_{0}^{L_{0}}\left[\left|u_{x}\right|^{2}+\left|\psi_{x}\right|^{2}\right] d x d t\right. \\
& \left.+\int_{0}^{T} \int_{L_{0}}^{L}\left[\left|v_{x}\right|^{2}+\left|\phi_{x}\right|^{2}\right] d x d t\right\} .
\end{aligned}
$$

If we choose and fix $\eta<C_{0}$ and $N_{3}$ such that $N_{3} C_{1}>C_{\eta}$, we get

$$
\mathcal{E}(T)-\mathcal{E}(0) \leq-C_{2} \int_{0}^{T} E(t) d t .
$$

Since $E(t)$ decreases, we have

$$
T E(T) \leq \int_{0}^{T} E(t) d t
$$


Using (5.2) in (5.1) we obtain

$$
\mathcal{E}(T)-\mathcal{E}(0) \leq-T C_{2} E(T) .
$$

Now observe that for sufficiently large $N$ we have

$$
\frac{N}{2} E(t) \leq \mathcal{E}(t) \leq 2 N E(t)
$$

from what follows that

$$
\mathcal{E}(T)-\mathcal{E}(0) \leq-\frac{C_{2}}{2 N} T \mathcal{E}(T),
$$

or else

$$
\mathcal{E}(T) \leq \alpha \mathcal{E}(0) \quad \text { with } \quad \alpha=\left[1+\frac{C_{2}}{2 N}\right]^{-1} .
$$

Note that $\alpha$ does not depend on the initial data, and hence, by using the semigroup property we have

$$
\mathcal{E}(t+T) \leq \alpha \mathcal{E}(t) \text { for every } t>0 .
$$

For $t>0$, there exists a natural $n$ and a real $r, 0 \leq r<T$ such that $t=n T+r$. This is equivalent to

$$
n=\frac{t}{T}-\frac{r}{T}
$$

Now, using the inequalities (5.3) and (5.4) $n$ times we obtain

$$
\begin{aligned}
\mathcal{E}(t) & \leq \alpha^{n} \mathcal{E}(r) \\
& \leq 2 N \alpha^{n} E(r) .
\end{aligned}
$$

Observing once more that $E(t)$ decreases we have

$$
\begin{aligned}
\mathcal{E}(t) & \leq 2 N \alpha^{\left(\frac{t}{T}-\frac{r}{T}\right)} E(0) \\
& \leq 2 N \alpha^{-1} E(0) e^{-w t},
\end{aligned}
$$

where $w=-\ln \left(\alpha^{\frac{1}{T}}\right)$.

Finally, using (5.3) we obtain

$$
E(t) \leq 4 \alpha^{-1} E(0) e^{-w t}
$$

and conclude the proof.

We can extend the previous theorem to the weak solutions by using simple density argument and the laws of semi-continuity for the energy functional. In this direction we have the following corollary. 
Corollary 1. Under the hypothesis of the previous theorem, there exist positive constants $C$ and $w$, such that

$$
E(t) \leq C E(0) e^{-w t}
$$

for every weak solution $(u, \psi, v, \phi)$ of the system (2.1)-(2.6).

\section{Concluding remarks}

During the past several decades, many authors have studied the same physical phenomenon for the Timoshenko system formulated into different mathematical models. Our approach to this problem is important not only from mathematical but mainly from the physical point of view with applications in Mechanics, amongst other branches of science. The system studied here is a model for vibrating beams subjected to two frictional mechanisms. More precisely, we proved that the presence of two frictional damping acting in a natural way on a small part of the beam, is enough to stabilize the whole beam. Moreover, it stabilizes quickly (at exponential rate). To the best of our knowledge, our result is the first in this direction. In this sense, this work generalizes the results previously obtained for Timoshenko's system where attrition acting in the whole beam was considered.

\section{REFERENCES}

[1] R.A. Adams, Sobolev Spaces, Academic Press, New York, (1975).

[2] F. Ammar-Khodja, A. Benabdallah, J.E.M. Rivera and R. Racke, Energy decay for Timoshenko systems of memory type. J. Differential Equations, 194(1) (2003), 82-115.

[3] R. Dautray and J.L. Lions, Analyse mathématique et calcul numérique pour les sciences et les techniques, Vol. 1, Masson S.A., Paris (1984).

[4] G.H. Hardy, J.E. Littlewood and G. Polya, "A Theorem of W. H. Young” $§ 8.3$ in Inequalities, 2nd ed., Cambridge University Press, Cambridge, (1988), pp. 198-200.

[5] J.U. Kim, A Boundary Thin Obstacle Problem for a Wave Equation. Comm. Partial Differential Equations, 14 (8 \& 9) (1989), 1011-1026.

[6] Z. Liu and C. Peng, Exponential stability of a viscoelastic Timoshenko Beam. Adv. Math. Sci. Appl., 8 (1998), 343-351.

[7] C.A. Raposo, J. Ferreira, M.L. Santos and N.N.O. Castro, Exponential Stability for the Timoshenko System With Two Weak Damping. Appl. Math. Lett., 18 (2005), 535-541. 
[8] Dong-Hua Shi and De-Xing Feng, Exponential decay of Timoshenko beam with locally distributed feedback. IMA J. Math. Control Inform., 18 (2001), 395-403.

[9] Dong-Hua Shi, De-Xing Feng and Qing-Xu Yan, Feedback stabilization of rotating Timoshenko beam with adaptive gain. Int. J. Control, 74(3) (2001), 239-251.

[10] S.P. Timoshenko and J.M. Gere, Mechanics of Materials, D. Van Nostrand Company, Inc, New York (1972). 